\title{
Combustión con eXCESo de ENTALPía
}

\author{
HenRy Copete LÓPeZ*
}

\section{Resumen}

En este trabajo se realiza una revisión del tema relacionado con las características de la combustión utilizando aire normal altamente precalentado. La tecnología se ha utilizado en los últimos años para proporcionar un aumento en la eficiencia térmica y una disminución significativa en el consumo de combustible y emisiones contaminantes respecto a los sistemas de combustión convencionales. Se presenta el concepto de combustión con exceso de entalpía, las principales propiedades de combustión que se afectan por el precalentamiento de los reactivos, los sistemas más utilizados para precalentar el aire, las ventajas y desventajas de esta tecnología y se describen algunas técnicas computacionales empleadas para el estudio de este tipo de combustión.

\section{Palabras clave}

Exceso de entalpía, aire precalentado, recuperación de calor.

\section{Alostract}

This work is a review of the characteristics of the combustion using highly preheated normal air. The technology has been used in the last years to provide an increase in the thermal efficiency and a significant decrease in the consumption of fuel and polluting emissions regarding the conventional combustion systems.

* MSc. Ingeniero Mecánico. Grupo GITER. InSTITUTO TECNOLÓGICO METROPOLITANO. Docente Investigador. henrycopete@itm.edu.co. 
The combustion with enthalpy excess concept is presented, and also the main combustion properties that are affected by the reactive preheated, the systems more used to preheat the air, the advantages and disadvantages of this technology and some computational technical.

\section{Key words}

Enthalpy excess, air preheated, heat recovery. 


\section{INTRODUCCIÓN}

Actualmente, se ve en todo el mundo el interés por la utilización de tecnologías de calentamiento cada vez más eficientes que ayuden a disminuir el consumo de combustible e incrementen la eficiencia térmica de los procesos en aplicaciones industriales. Esto ha sido motivado por la incertidumbre en los precios de los combustibles fósiles, los pronósticos que hablan de limitadas reservas de petróleo en las décadas venideras, las políticas de producción más limpia con mínimos impactos ambientales y la necesidad de incrementar la competitividad para asegurar la permanencia en el mercado. Lo anterior ha conducido la investigación y el desarrollo tecnológico en sistemas que permitan recuperar el calor residual de los gases de escape para precalentar el aire de combustión. Se han venido desarrollando nuevas tecnologías como los recuperadores de calor, los quemadores porosos y los regeneradores de calor.

La utilización de las tecnologías de combustión con aire a alta temperatura influye sobre las características de la combustión ampliando los límites de inflamabilidad, incrementando la velocidad de combustión, aumentando la temperatura de llama y maximizando el calor disponible en los procesos (Getting, 1986). Estos efectos combinados mejoran la estabilidad de llama y la combustibilidad de gases de bajo poder calorífico (Zabetakis, 1965).

El aumento en las temperaturas de llama debido al precalentamiento del aire de combustión puede producir efectos indeseables por la formación de emisiones de óxidos nitrosos asociados a las altas temperaturas, por lo que también se han desarrollado técnicas que mitigan este efecto mediante la recirculación de gases de combustión en la zona de reacción (Gupta, 2006).

En este trabajo se realiza una revisión de las características de la combustión con aire normal $\left(21 \% \mathrm{O}_{2}-79 \% \mathrm{~N}_{2}\right)$ altamente precalentado, identificando las mejores tecnologías por emplear, los parámetros básicos de funcionamiento, ventajas, desventajas, perspectivas, técnicas de simulación y problemas asociados al fenómeno que permitan obtener fundamentos, teóricos, experimentales 
y tecnológicos, para tener herramientas que permitan en el país la investigación de esta tecnología, utilizando múltiples combustibles gaseosos como el metano, el propano, el biogás, el gas de síntesis y otras mezclas.

\section{Combustión con eXCESo de entalpía}

El término "exceso de entalpía" es usado para describir la entalpía entregada por los productos de combustión a los reactivos para incrementar su temperatura con efectos importantes sobre las velocidades de combustión, los límites de inflamabilidad y las temperaturas de llama (Weinberg, 1986), produciendo un incremento en la eficiencia de combustión, una disminución en las emisiones contaminantes y facilitando el quemado de combustibles de bajo poder calorífico.

La cantidad de energía durante el proceso de combustión está dada por la ecuación (1) (Takeno, 1979.):

$$
\int_{T_{0}}^{T_{f}} C_{p} d T=Q_{c}+Q_{a}=H_{f}-H_{0}
$$

Donde, $T_{f}$ es la temperatura final, $T_{0}$ la temperatura inicial, $Q_{c}$ el calor liberado por la conversión de energía química, $Q_{a}$ el calor sensible añadido con el precalentamiento del aire, $H_{f}$ y $H_{0}$ las entalpías en los dos estados.

La figura 1 ilustra el concepto de la combustión con exceso de entalpía mediante la recirculación de calor. El cambio de entalpía para la combustión normal sin precalentamiento del aire es indicado por la línea punteada. Debido a las pérdidas finitas de calor, la temperatura no puede alcanzar completamente los valores que daría una llama adiabática. Por otro lado, la variación de entalpía con la recuperación de calor es presentada por medio de la línea continua. El precalentamiento de los reactivos incrementa la entalpía permitiendo obtener temperaturas mayores a la de llama adiabática en condiciones normales (Tanaka, 2001). 
La temperatura de llama teórica, se puede decir que incrementa en forma lineal con el incremento de la temperatura de precalentamiento del aire de combustión (Zhu, 2004), como puede apreciarse en la figura 2 para la reacción de una mezcla propano $\left(\mathrm{C}_{3} \mathrm{H}_{8}\right)$ - gas de síntesis $\left(20 \% \mathrm{CO}, 20 \% \mathrm{H}_{2}, 4 \% \mathrm{CH}_{4} \mathrm{y} \mathrm{N}_{2}\right.$ balance $)$ y diferentes factores de aireación, obtenida a partir de la cinética química en fase gaseosa para una llama de premezcla propagada libremente (Copete, 2008).

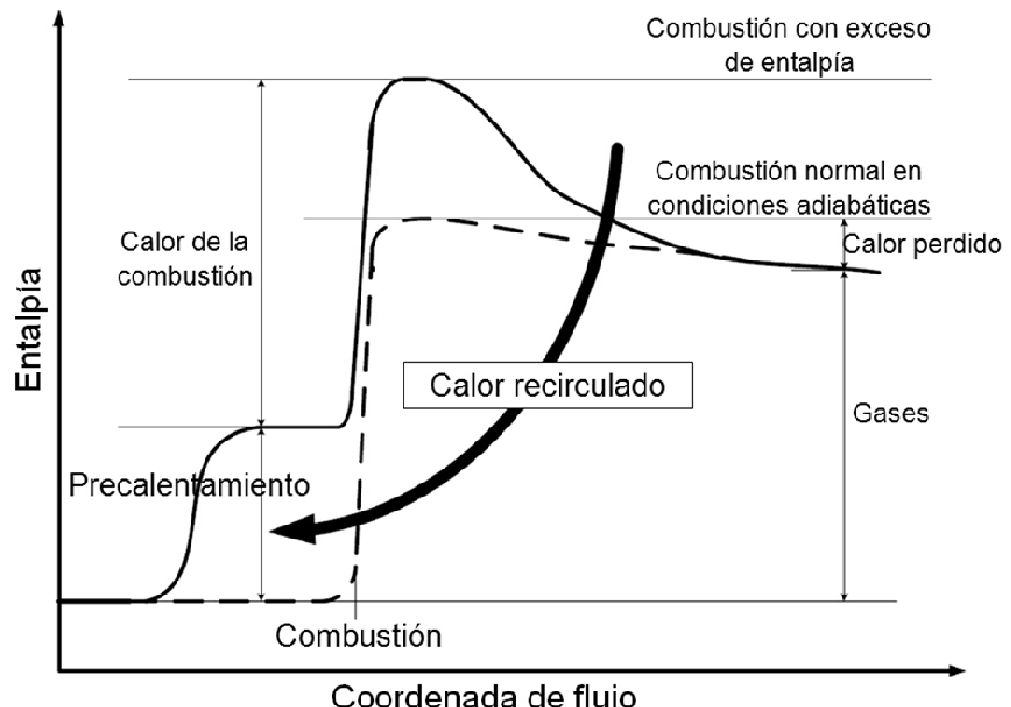

Figura 1. Concepto de exceso de entalpía. Adaptado de (Tanaka, 2001)

El exceso o defecto de aire también afecta la temperatura de llama teórica encontrando la más alta en la relación estequiométrica cuando $\mathrm{n}=1$ para cada temperatura de precalentamiento, disminuyendo notablemente cuando se incrementa el exceso o defecto de aire como puede apreciarse para $\mathrm{n}=0.5, \mathrm{n}=1.75$ $\mathrm{y} \mathrm{n}=2$. 


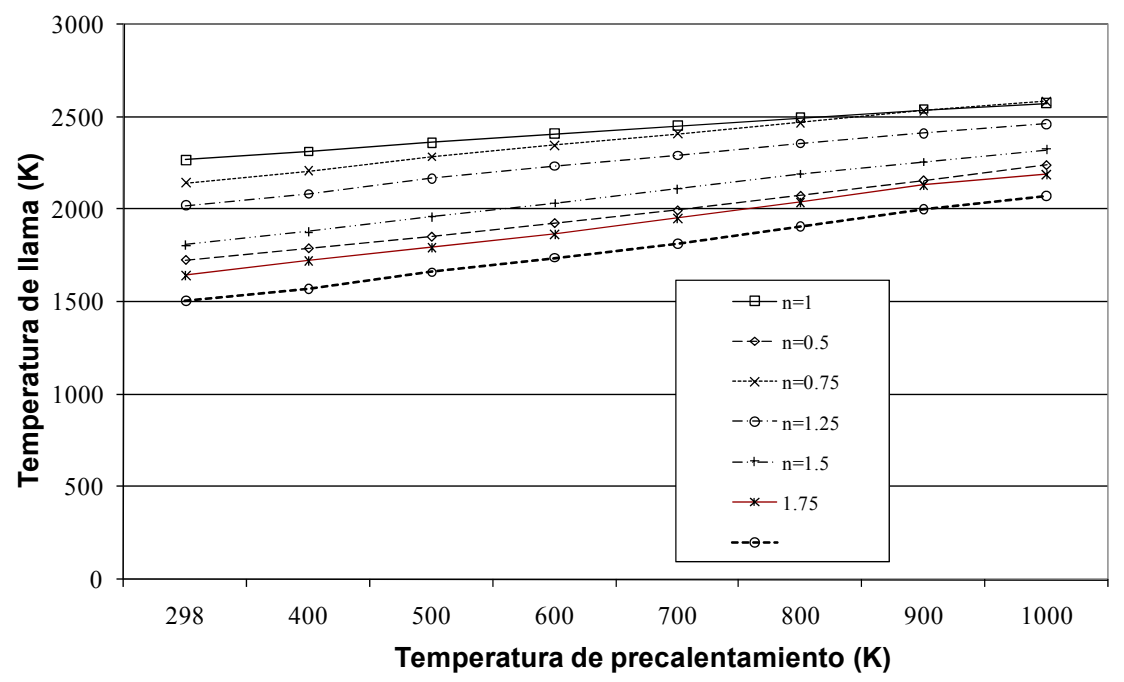

Figura 2. TEMPERATURA DE LLAMA TEÓRICA EN FUNCIÓN DE LA TEMPERATURA DE PRECALENTAMIENTO DEL AIRE PARA DIFERENTES FACTORES DE AIREACIÓN

\section{Sistemas para precalentar el aIRE DE COMbustión}

Una combustión eficiente puede ser lo suficientemente alta en muchas aplicaciones industriales si se controla la relación entre el aire y el combustible, sin embargo, la eficiencia útil no siempre es alta y para aumentarla se ha venido trabajando con la combustión con exceso de entalpía utilizando tres principales tecnologías: los recuperadores o intercambiadores de calor gas-gas, y los intercambiadores de calor que utilizan una matriz sólida como los regeneradores de calor y los quemadores porosos.

Se han sugerido en la literatura un gran número de esquemas para la realización práctica de la combustión con exceso de entalpía mediante intercambiadores de calor gas-gas, dos ejemplos son los ilustrados en la figura 3. 


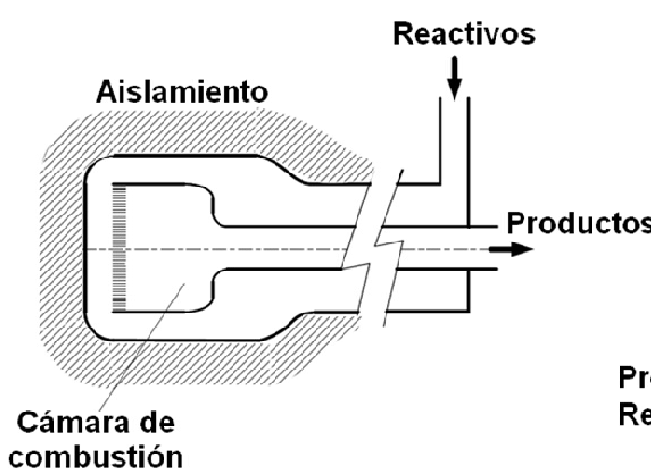

a)

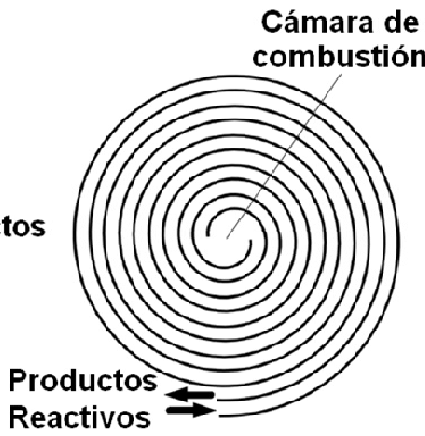

b)

Figura 3. Quemadores de CAlor ReCIRCULAdo. A) Quemador con INTERCAMbiador A CONTRAFLUJO Y B) QUEMADOR CON INTERCAMBIADOR EN ESPIRAL DE DOS PASOS. AdAPTADO DE (WOOD, 2008)

En experimentos usando quemadores de doble espiral, se han reportado combustión estable para concentraciones de metano de hasta $1.6 \%$ cuando el límite inferior de inflamabilidad es del orden del 5\% (Wood, 2008). Estudios con este tipo de sistemas se siguen realizando por los beneficios presentados en la estabilidad de la combustión, aún en la escala de la microcombustión, en la cual se ha podido obtener una combustión en modo sin llama (Sitzki, 2001).

Otra alternativa interesante para alcanzar una combustión con exceso de entalpía consiste en la incorporación de un sólido poroso con grandes propiedades de transferencia de calor a la zona de reacción, lo cual produce una autorecuperación interna de calor dándose la combustión al interior del mismo intercambiador que es la idea básica de un quemador poroso.

La primera demostración analítica de esta tecnología fue realizada por (Takeno, 1979) y comprobada experimentalmente más adelante (Kotani, 1982) y (Behbahani, 1984). Este método de recuperación de calor incorpora los tres modos de transferencia de calor: conducción, convección y radiación como se muestra en las figuras 4 y 5a) (Wood, 2008). El proceso puede resumirse de la siguiente manera: aguas abajo de la zona de reacción el gas está 
más caliente que el sólido y por tanto el calor es transferido por convección desde los productos de combustión a la matriz porosa. $\mathrm{El}$ sólido caliente conduce y radia calor en la dirección aguas arriba del quemador; antes de la zona de reacción el sólido alcanza una temperatura mayor a la de la mezcla de reactivos y de esta forma transfiere calor por convección a la corriente. Los gases entrantes son entonces precalentados hasta que alcanzan la temperatura de ignición, la reacción toma lugar y el ciclo continúa. Los perfiles de temperatura en el sólido y el gas son como los que se muestran en la figura 5b. (Barra, 2004) reporta que mediante estos sistemas es posible recuperar hasta un $25 \%$ de la energía que entra con el combustible.

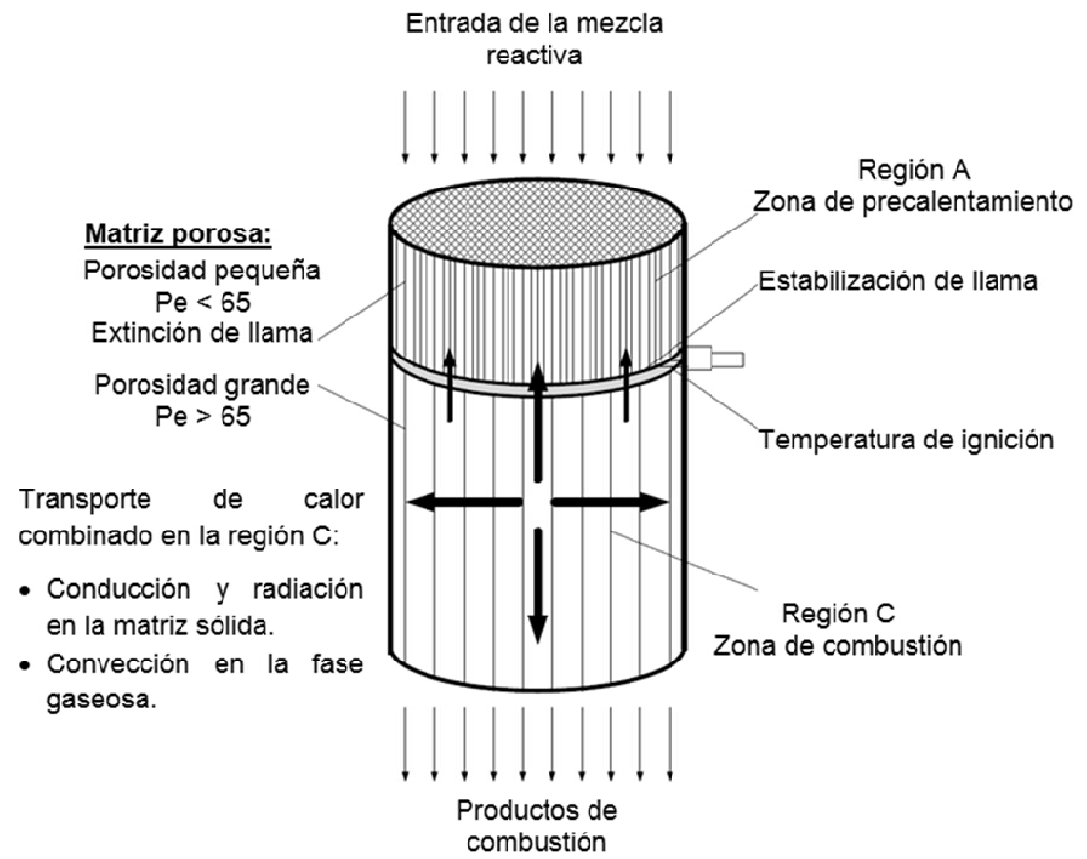

Figura 4. Proceso de transferencia de calor en un QUemador poroso.

Adaptado de (Delalic, 2004) 
a)

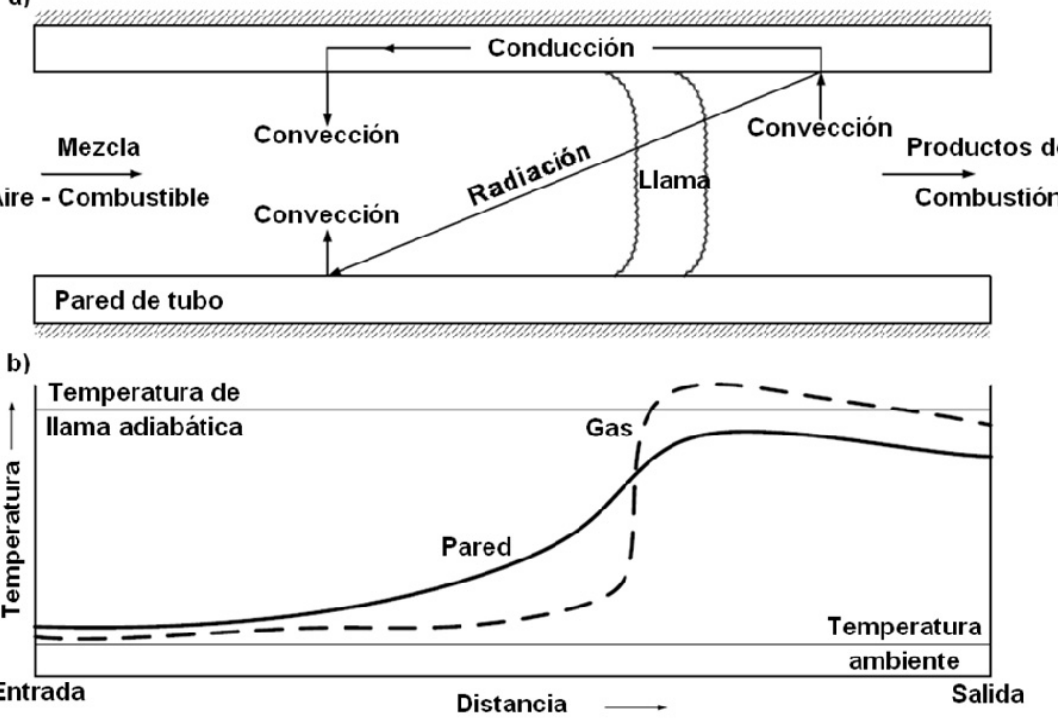

Figura 5. A) REPRESENTACIÓN ESQUEMÁticA DE LA TRANSFERENCIA DE CALOR EN UN QUEMADOR POROSO Y B) PERFIL DE TEMPERATURA DEL GAS Y EL SÓLIDO. AdAPTADO DE (WoOd, 2008)

Los materiales más importantes usados en esta tecnología son espumas cerámicas construidas normalmente de $\mathrm{ZrO}_{2}, \mathrm{SiC}_{\mathrm{C}} \mathrm{Al}_{2} \mathrm{O}_{3}$ y su porosidad varía entre la zona de precalentamiento donde no hay reacción química y la zona de combustión. A nivel experimental se ha podido demostrar mediante el número modificado de Péclet la condición para que exista propagación de la llama (Mößbauer, 1999).

$$
P s=\frac{S_{L} d_{m} C p \rho}{k} \geq 65
$$

Donde $S_{L}$ es la velocidad laminar de combustión, $d_{m}$ el diámetro equivalente del poro, $C_{p}$ el calor específico, $\rho$ la densidad y $k$ la conductividad de la mezcla gaseosa.

Las principales ventajas de esta tecnología es que son sistemas pequeños y compactos, de muy alta variación de potencia y mínimas 
emisiones contaminantes independiente de la carga, además de que pueden ser aplicados en muchos procesos industriales, a nivel residencial, para calentamiento de aire, en turbinas a gas, en generadores de vapor y calentamiento de fluidos, tubos radiantes, entre otros.

Para procesos industriales a alta temperatura, los regeneradores de calor se encuentran como la tecnología más conveniente para recuperar el calor residual de los productos de combustión. Son dos intercambiadores de calor acoplados cada uno a un quemador para trabajar alternadamente, mientras un quemador está en la fase de combustión se está almacenando calor en el regenerador del quemador opuesto. El calor transferido al refractario de los gases calientes es almacenado y posteriormente entregado al aire de combustión, el cual de esta manera es precalentado aumentando su energía interna antes de entrar al quemador de gas (Amell, 2006). Aproximadamente, un $85 \%$ del calor residual puede ser recuperado mediante esta tecnología (NEDO, 2006). En la figura 6 se presenta esquemáticamente el funcionamiento descrito.

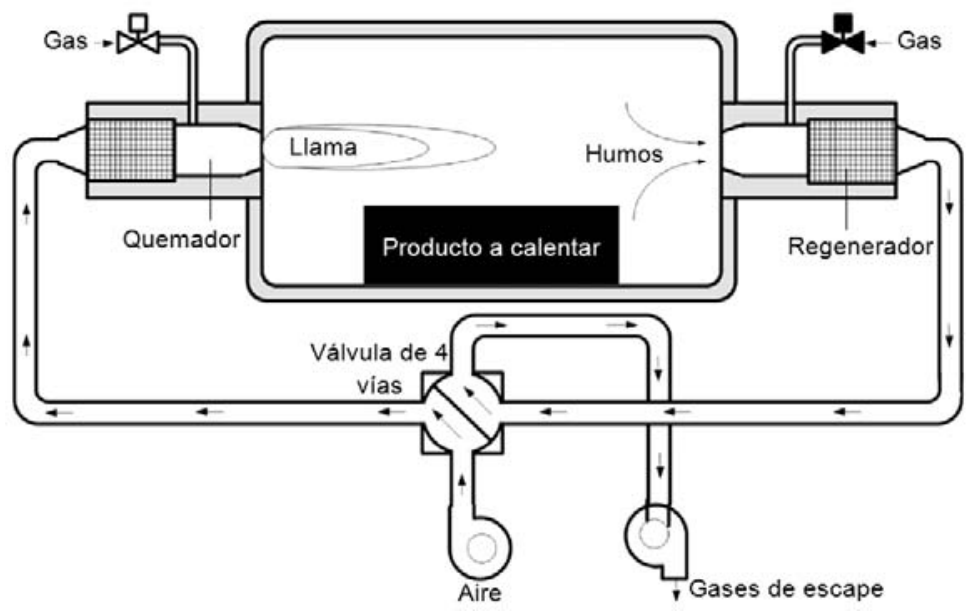

Figura 6. Sistemas de COMBUStión REgenerativos 
En 1858, William Siemens construyó el primer horno experimental con regeneradores para precalentar el aire de combustión, con el objetivo de incrementar la temperatura de llama, dos años después construyó y patentó el primer horno comercial con recuperación regenerativa de calor aplicable a la fundición de vidrio y escalado a través de la historia a muchos otros procesos industriales. Las primeras generaciones de esta tecnología se caracterizaban por ser sistemas robustos ubicados al exterior de las cámaras de combustión que resultaban de gran tamaño y altos costos de inversión (Baukal, 2004).

A partir de los años 1980 comenzaron los trabajos para desarrollar quemadores regenerativos más compactos mediante la incorporación de la recuperación de calor a la cámara de combustión, a través de arreglos compactos y de gran efectividad de transferencia de calor que se conocen como sistemas autorregenerativos. Estos sistemas regenerativos compactos poseen una efectividad cercana al 90\%, alcanzando altos niveles de precalentamiento del aire que pueden estar en un $85 \%$ de la temperatura del proceso, mejorando la uniformidad de la temperatura en el horno, incrementando la productividad y ahorrando cantidades considerables de combustible (Yu, 2002).

Diferentes materiales han sido empleados como regeneradores térmicos, estos deben cumplir un excelente desempeño a altas temperaturas, resistencia a los choques térmicos y ataques químicos. Por esta razón se utilizan materiales cerámicos compuestos por $\mathrm{Al}_{2} \mathrm{O}_{3} / \mathrm{SiO}_{2}$, que se pueden encontrar en diferentes configuraciones geométricas. La figura 7 muestra las usualmente más utilizadas: esferas y una configuración en panal de abeja "honeycomb" que brinda menores caídas de presión y brinda una mayor área de transferencia de calor por unidad de volumen (Suzukawa, 1997). 

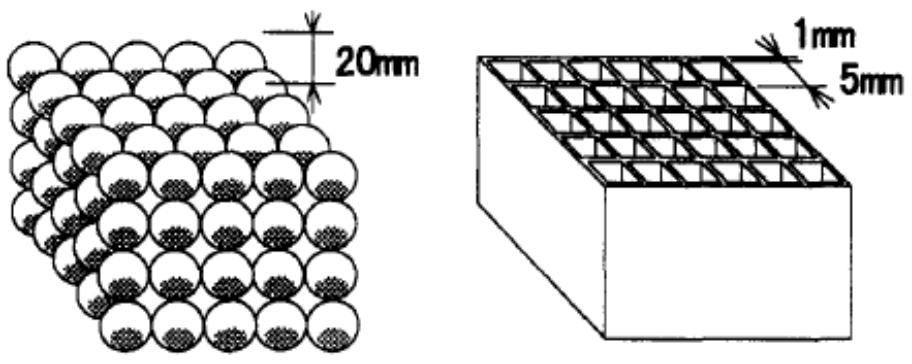

Figura 7. Sistemas de combustión Regenerativo. Adaptado de (SuzukaWa, 1997)

\section{ACERCA DE LOS LÍMITES DE INFLAMABILIDAD, LAS EMISIONES CONTAMINANTES Y LA EFICIENCIA TÉRMICA}

Los límites de inflamabilidad de una mezcla aire-combustible se definen como las relaciones máximas y mínimas en porcentaje entre el volumen de gas y el volumen de la mezcla aire-gas para la cual esta mezcla es inflamable y puede auto-propagarse, significa que una mezcla cuya composición no se halle dentro de los límites de inflamabilidad no puede encenderse aunque esté expuesta a una chispa o a una llama. Porque el límite inferior de inflamabilidad de una mezcla aire/gas disminuye cuando se incrementa la temperatura inicial de la mezcla de acuerdo con la ley de Burgess-Wheeler (Zabetakis, 1965), la combustión con aire precalentado puede dirigir a una reducción del límite inferior de inflamabilidad.

$$
\text { L.I.I }{ }_{T}=\text { L.I.I }_{298}\left[1-\frac{3.14(T-298)}{\text { L.I.I }{ }_{298} P C I}\right]
$$

Donde L.I.I $I_{T}$ es el límite inferior de inflamabilidad a la temperatura de precalentamiento de los reactivos (en porcentaje), y $P C I$ el poder calorífico inferior (en $\mathrm{MJ} / \mathrm{kmol}$ ).

Por otro lado, el límite superior de inflamabilidad tiende a aumentarse al incrementar la temperatura de precalentamiento del aire lo cual genera, entonces, una ampliación significativa del 
intervalo de inflamabilidad si se compara con el intervalo de inflamabilidad a temperatura ambiente como se ilustra en la figura 8 . Un gran incremento en la temperatura puede causar autoignición, además, si se trabaja con un combustible de bajo poder calorífico, la región de inflamabilidad desaparece a temperatura ambiente y reaparece cuando la mezcla es precalentada por encima de ciertos niveles de temperatura (Yeganeh, 2005).

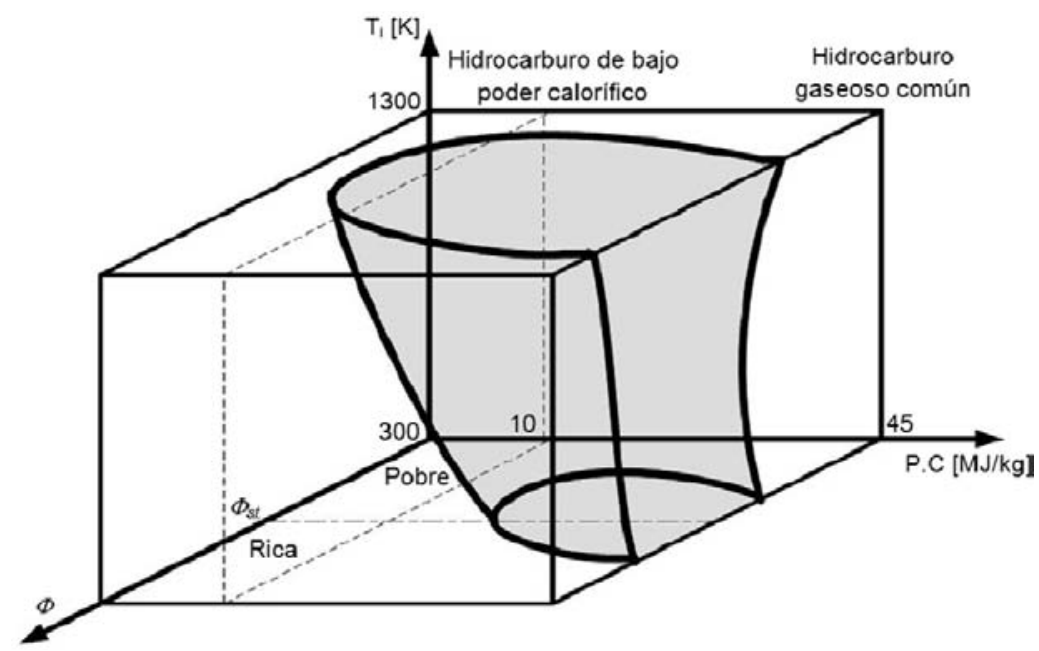

Figura 8. Región INFLAMABle EN FUnCIÓN DEL PODER CALORIFICO DEL COMBUSTIBLE, LA TEMPERATURA INICIAL DE LA MEZCLA Y LA RELACIÓN DE EQUIVALENCIA.

ADAPTADO DE (MASASHI, 1998)

Como ya se ha mencionado, la temperatura de precalentamiento del aire trae consigo un incremento en los picos de temperatura de llama, lo cual desde el punto de vista de la formación de NOx térmicos genera mayores concentraciones de este gas de efecto invernadero (Seepana, 2006), con una tendencia, como la mostrada en la figura 9 . 


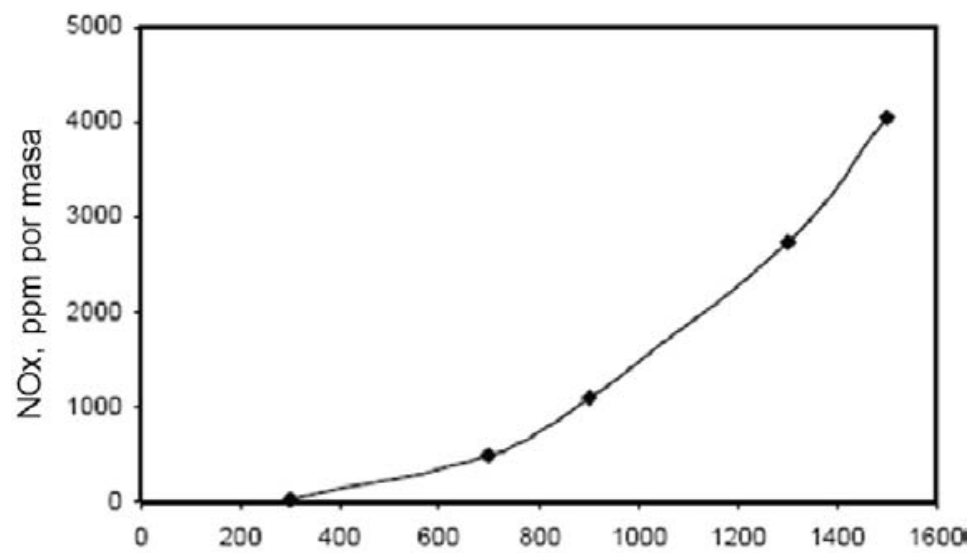

Figura 9. EMISIONES de NOx EN FUnCIÓN DE LA TEMPERATURA DE PRECALENTAMIENTO DE AIRE

Para atenuar y controlar estos incrementos en las emisiones de NOx, se han desarrollado diferentes técnicas como la combustión por etapas, el reburning, flame cooling, combustión con premezcla pobre y una muy importante, la recirculación de los productos de combustión en la zona de reacción lo que genera un viciamiento local del aire, reduce las temperaturas de llama y por ende las emisiones de NOx (Masashi, 1998). Mediante la recirculación de humos se pueden obtener concentraciones muy por debajo del $21 \%$ de $\mathrm{O}_{2}$ en la zona de reacción y con el precalentamiento del comburente por encima de la temperatura de autoignición del combustible, es posible obtener el fenómeno de combustión sin llama conocido como "flameless".

Es bien conocido en el mundo, el rol del $\mathrm{CO}_{2}$ emitido por la combustión de combustibles fósiles sobre el fenómeno del calentamiento global (Cavaliere, 2004). Al sustituir sistemas de combustión convencionales por tecnologías con aire precalentado se logra reducir las emisiones $\mathrm{CO}_{2}$ debido al incremento de la eficiencia de combustión y la reducción del consumo de combustible. En la figura 10 se puede apreciar el posible ahorro en combustible para un proceso de calentamiento con el aumento de la temperatura de 
precalentamiento del aire para diferentes temperaturas de gases evacuados, usando Fuel Oil como combustible y un exceso de aire del 20\% (NEDO, 2006).

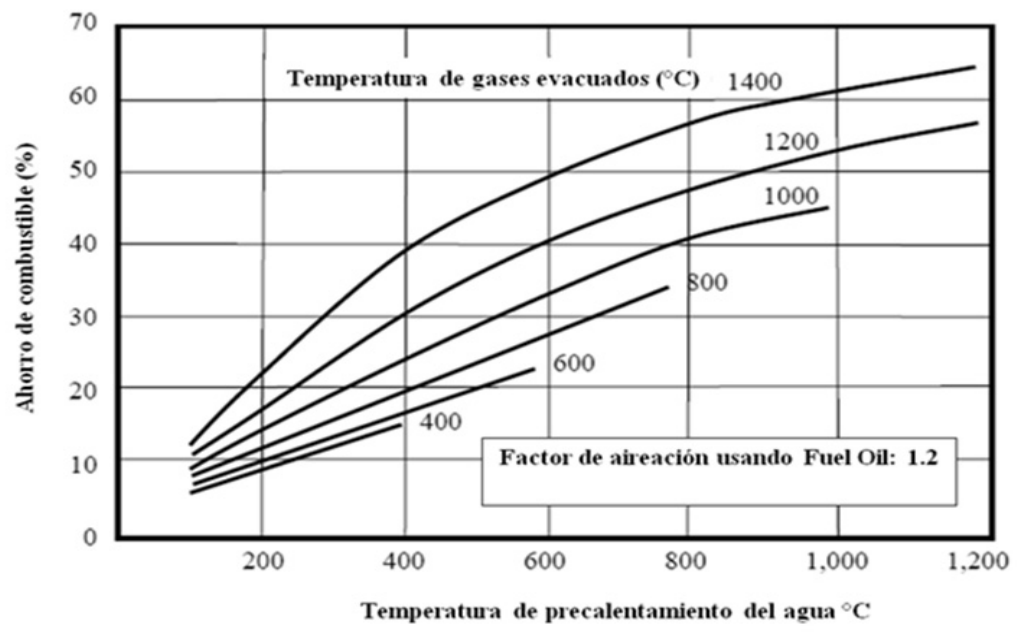

Figura 10. AHORRo de COMBUStible EN FUnCIÓN DE LA TEMPERATURA de PRECAlentamiento del aire. Adaptado de (Nedo, 2006)

\section{Modelado y Simulación DE LA COMBUStión}

En el pasado, las investigaciones en combustión se llevaban a cabo por medio de métodos empíricos que resultaban costosos y demandaban gran cantidad de tiempo. Con los recientes avances en métodos numéricos y los grandes potenciales de la computación, esta dependencia se ha trasladado hacia la simulación numérica de los fenómenos.

La dinámica de fluidos computacional puede ser usada como una herramienta práctica de diseño para estudiar nuevas configuraciones de sistemas térmicos con diferentes condiciones de operación. El potencial de estos métodos numéricos generalmente ha sido probado y validado con resultados experimentales, según sea el caso. 
Tives en 1998 realizó un trabajo en un horno regenerativo con precalentamiento de aire para validar diferentes modelos del código FLUENT en una combustión con llama de difusión. Se emplearon un total de 14 casos de los cuales sólo 11 alcanzaron una convergencia, y éstos fueron comparados con resultados experimentales. Los tres mejores casos en términos de aproximación y esfuerzo computacional fueron los que emplearon el modelo $k-\varepsilon$ para turbulencia, PDF - Mixture Fraction para la combustión y un coeficiente de absorción constante en la radiación (Tives, 1998).

Tabacco et al. simularon una cámara de combustión sin llama, aplicando dos modelos de combustión: Finite Rate/Eddy Dissipation (Magnussen \& Hjertager), usando un mecanismo de reacción de un paso (mecanismo de Westbrook \& Dryer) y el modelo PDF/mixture fraction suponiendo equilibrio químico. Ambos modelos dieron buenos ajustes cualitativos de los datos experimentales (Tabacco, 2002).

Para la aplicación de la simulación numérica y sus modelos matemáticos en combustión con aire altamente precalentado, Weihok y Blasiak propusieron una metodología para seguir que puede ser resumida como (Weihong, 2006):

- Estudiar los modelos disponibles en los programas computacionales, desarrollar nuevos modelos según requerimientos y verificar los modelos usados.

- Producir información del campo de estudio, esto es: campos de velocidad, distribución de temperatura, niveles de concentración de especies, distribución de la transferencia de calor, niveles de quemado, gradientes de presión, etc.

- Comparar y validar los resultados de la simulación con resultados experimentales.

- Análisis de las características principales de los resultados como la combustión, fluidodinámica y estructura de la llama.

- Estudiar la influencia de diferentes parámetros sobre la combustión, las emisiones contaminantes y la transferencia de calor. Estos parámetros incluyen la concentración de $\mathrm{O}_{2}$ en la zona de reacción, la temperatura de precalentamiento del 
aire de combustión, la temperatura del combustible, los flujos de aire y combustible y la localización de la llama.

Otra herramienta computacional importante por utilizar en el estudio de la combustión con aire altamente precalentado es el software comercial CHEMKIN que permite resolver e interpretar la combustión a partir de la cinética química en fase gaseosa. Zhu et al. lo utilizaron para estudiar el efecto de la temperatura de precalentamiento del aire sobre las emisiones de NOx y la formación de soot para diferentes concentraciones de $\mathrm{O}_{2}$ y diferentes concentraciones del combustible (Zhu, 2004), con otros estudios se han podido predecir las concentraciones de especies químicas en el equilibrio químico, calcular las temperaturas de llama teórica y las velocidades de combustión.

Utilizando este mismo software, Barra et al. realizaron un estudio numérico sobre el efecto de las propiedades del material sobre la estabilización de llama en un quemador poroso. En la figura 11 se presenta el comportamiento de la temperatura del gas, del sólido y el calor liberado en el quemador poroso (Barra, 2003), en la interface es donde se estabiliza la llama y como puede observarse se obtiene una temperatura de precalentamiento de la mezcla cercana a 1200 K. En este caso, el software permitió analizar diferentes porosidades y propiedades del material encontrando los límites de estabilización de la llama para diferentes factores de aireación y las velocidades óptimas de combustión.

El comportamiento de la velocidad de deflagración laminar calculada en CHEMKIN se presenta en la figura 12 para la mezcla Propano-Syngas, en las cuales se puede observar su incremento a medida que se aumenta la temperatura de precalentamiento del aire. Esta velocidad de deflagración también se ve afectada por la relación aire-combustible, con los máximos valores cuando se cuenta con una relación estequiométrica, es decir con un factor de aireación $\mathrm{n}=1$. Este aumento en la velocidad de deflagración puede afectar la estabilidad de combustión posibilitando el desprendimiento de la llama, por lo cual dependiendo del sistema de combustión deben ser tenidas en cuenta diferentes técnicas de estabilización de la llama. 


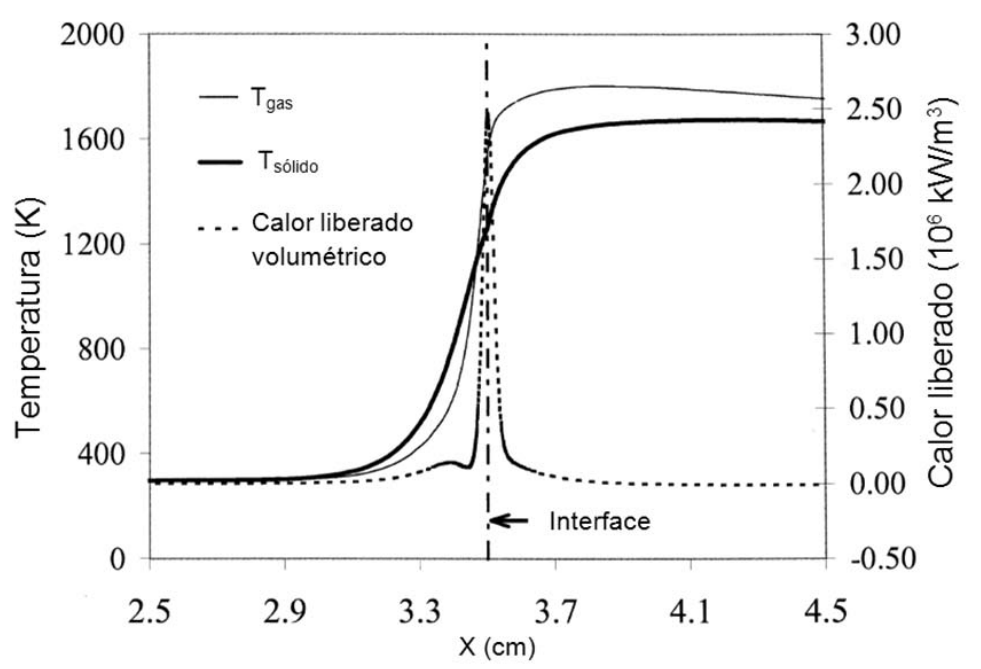

Figura 11. Temperatura del Gas, Sólido y CALOR LIBERADO EN UN QUEMADOR POROSO. AdAPTADO DE (BARRA, 2003)

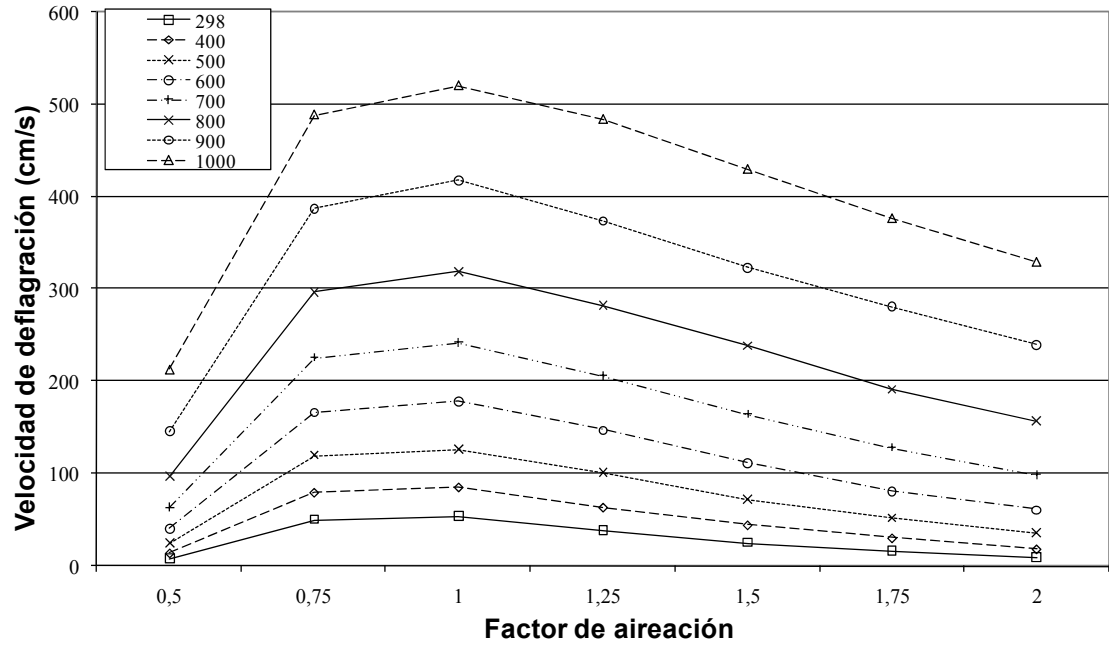

FIgURA 12. Velocidad de COMBUStIÓN LAMINAR EN FUNCIÓN DE LA TEMPERATURA DE PRECALENTAMIENTO DE AIRE Y DIFERENTES FACTORES DE AIREACIÓN 


\section{Conclusiones}

- El precalentamiento del aire tiene efectos sobre diferentes propiedades de combustión: disminuye el límite inferior de inflamabilidad y aumenta el límite superior, es decir que se amplía el intervalo de inflamabilidad, lo que hace posible el quemado de combustibles de bajo poder calorífico y concentraciones por fuera del intervalo a temperatura ambiente. La temperatura de llama teórica se incrementa en forma lineal con el aumento de la temperatura de precalentamiento de aire. La velocidad de combustión también es incrementada notablemente.

- El incremento de la temperatura de llama trae consigo un incremento en las emisiones de NOx, por lo que se han desarrollado diferentes técnicas para mitigar este efecto, la más importante quizás es la recirculación de productos de combustión que disminuye la concentración de $\mathrm{O}_{2}$ en la zona de reactivos, proceso conocido como combustión flameless, con un sin número de investigaciones a nivel mundial en este tema.

- Las principales tecnologías para precalentar el aire de combustión son los recuperadores gas-gas, los regeneradores de calor gas-sólido-gas y los quemadores porosos, con ventajas de unos frente a los otros según sea el campo de aplicación.

- Existen comercialmente diferentes herramientas computacionales que permiten mediante la aplicación de métodos numéricos simular el comportamiento de la combustión, y los fenómenos fluidodinámicos y térmicos; entre los más importantes se tiene el codigo CFD FLUENT y el software CHEMKIN para predecir la combustión a partir de la cinética química en fase gaseosa.

- Identificados y descritos los avances, tendencias y bondades que brinda la tecnología de combustión con exceso de entalpía, el autor de este trabajo cree totalmente pertinente para la región y el país la apropiación y desarrollo tecnológico en esta temática mediante proyectos de investigación que permitan incrementar eficiencia térmica en diferentes procesos industriales y la 
combustión y utilización de gases de bajo poder calorífico como el gas de síntesis o el biogás, actualmente desechado en muchos biodigestores y rellenos sanitarios.

\section{Bibliografía}

Amell A., Copete H. y Cadavid F. (2006). Desarrollo y evaluación de un sistema de combustión autorregenerativo y radiante. Revista Dyna. Año 74, $\mathrm{N}^{\circ} 151,61-69$.

Barra A., Ellzey J. (2004). Heat recirculation and heat transfer in porous burners. Combustion and Flame 137, 230-241.

Barra A., Diepvens G., Ellzey J y Henneke M. (2003). Numerical study of the effects of material properties on flame stabilization in a porous burner. Combustion and Flame 134, 369-379.

Baukal C. (2004). Industrial Burner Handbook. CRC Press Boca Raton New York, 413-440.

Behbahani H., Kotani Y., Takeno T. (1984). An excess enthalpy flame combustor for extended flow ranges. Proceedings of the Twentieth Symposium International on Combustion.

Cavaliere A. y Joannon M. (2004). Mild Combustion. Progress in Energy and Combustion Science 30, 329-366.

Copete H. (2008). Estudio del efecto de la temperatura de precalentamiento de aire y el factor de aireación sobre la temperatura de llama, la velocidad laminar de combustión y la concentración de especies en la combustión de la mezcla Propano-Syngas. Documento de trabajo. Doctorado en Ingeniería. Universidad de Antioquia.

Delalic N., Mulahasanovic D y Ganic E. (2004). Porous media compact heat exchanger unit-experiment and analysis. Experimental Thermal and Fluid Science 28, 185-192.

Getting M. (1986). The regenerative burner in the metal industry. Gas Warme International, 292-296.

Gupta A. (2006). Clean power generation from fuels using high temperature air combustion technology. The $2^{\text {nd }}$ Joint International Conference on "Sustainable Energy and Environment", 11-20.

Kotani Y., Takeno T. (1982). An experimental study on stability and combustion characteristics of an excess enthalpy flame. Proceedings of the Nineteenth Symposium International on Combustion. 
Masashi K. y Hasegawa T. (1998). The science and technology of combustion in highly preheated air. The twenty-seventh symposium (International) on combustion - The Combustion Institute.

Mößbauer S. Pickenäcker O., Pickenäcker K. y Trimis D. (1999). Applications of the porous burner technology. Fifth International Conference on Technologies and Combustion for a Clean Environment (Clean Air V), Lisbon (Portugal), 519-526.

NEDO. (2006). Japanese Technologies for Energy Savings/GHG Emissions Reductions. NEDO, 29-56.

Seepana S. y Jayanti S. (2006). Prediction of HITAC features in a pilot furnace. Advances in Energy Research, 180-205.

Sitzki L., Borer K., Schuster E. y Ronney P. (2001). Combustion in Microscale Heat-Recirculating Burners. The Third Asia - Pacific Conference on Combustion, 24-27.

Suzukawa Y., Sugiyama S., Hino Y, Ishioka M. y Mori I. (1997). Heat transfer improvement and NOx reduction by highly preheated air combustion. Energy Convers. Mgmt, 1061-1071.

Tabacco D., Innarella C., y Bruno C. (2002). Theoretical and numerical investigation on flameless combustion. Combustion Science and Technology, 1-35.

Takeno T., Sato K. (1979.). An excess entalphy flame theory. Combustion Science Technology 20, 73-84.

Tanaka R., Shinoda M. y Arai N. (2001). Combustion characteristics of a heat recirculating ceramic burner using a low calorific fuel. Energy Conversion and Management 42, 1897-1907.

Tives N. (1998). Numerical simulations of turbulent non-premixed combustion in a regenerative furnace. National Library of Canada, 1272-1281.

Weihong Y. y Blasiak W. (2006). CFD as applied to high temperature air combustion in industrial furnaces. IFRF Combustion Journal, 23-33.

Weinberg F. (1986). Combustion in heat-recirculation burners. Advanced Combustión Methods, academics Press, 930-945.

Wood S., y Harris A. (2008). Porous burner for lean - burn applications. Progress in Energy and Combustion Science 34, 667-684.

Yeganeh M. (2005). Effects of preheated combustion air on laminar coflow diffusion flames under normal and microgravity conditions. University of Maryland, 265-268. 
Yu J., Zhang M., Fan W., Zhou Y. y Zhao G. (2002). Study on performance of the ball packed - bed regenerator: experiments and simulations. Applied Thermal Engineering 22, 641-651.

Zabetakis M. (1965). Flammability characteristics of gases and vapors. Bureau of mines bulletin, 627-647.

Zhu T., Zheng, W y Cao, Z. (2004). Numerical study on NOx and soot emission in high temperature air combustión based on detailed chemical kinetics. Tongji University Shangai. 